\title{
Fractais no Laboratório Didático
}

(Fractals in the didactic Laboratory)

\author{
M. Amaku†, M. Morallesł, L. B. Horodynski-Matsushigue† e P. R. Pascholati† \\ $\dagger$ Instituto de Física da Universidade de São Paulo \\ Caixa Postal 66318, CEP 05 315-970, São Paulo, SP, Brasil \\ $\ddagger$ Instituto de Pesquisas Energéticas e Nucleares - Comissão Nacional de Energia Nuclear \\ Caixa Postal 11049, CEP 05 508-900, São Paulo, SP, Brasil \\ E-mail: lighia@if.usp.br
}

Recebido em 19 de Abril de 2001. Aceito em 27 de Novembro de 2001.

\begin{abstract}
É discutida a conceituação de objetos com estrutura fractal, tendo em vista sua utilização em experiências motivadoras no laboratório didático. Foram estudadas bolas de pão amassado, que podem ser empregadas como contraponto não fractal $(D \sim 3,0)$ às bolas de papel amassado, as quais apresentam dimensão fractal $(D \sim 2,5)$, salientando a necessidade de um cálculo correto de incertezas. Três tipos de pão foram investigados: dois brancos, de forma e italiano, e um preto denso, do tipo alemão de três grãos. Desses, apenas o pão branco italiano demonstrou possível estrutura fractal, após o amassamento, caracterizada pela dimensão fractal $D=2,81 \pm 0,06$, enquanto os resultados obtidos para os pães preto e de forma, $D=2,95 \pm 0,06$ e $D=2,98 \pm 0,04$ respectivamente, não os tipificam como fractais. São analisados os problemas conceituais que resultaram em dimensão fractal 2,08 $\pm 0,25$ para o pão, em informação encontrada na literatura de escopo didático.
\end{abstract}

\begin{abstract}
The concept of objects with a fractal structure is discussed, in the context of their use in motivating activities for the didactic Laboratory. Spheres of squashed bread, which may be employed as nonfractal counterexample $(D \sim 3,0)$ to the fractal crumpled paper balls $(D \sim 2,5)$, were analysed and the need for correct computation of the uncertainties is stressed. Three kinds of bread were investigated: two white ones, an usual sandwich and an italian type, and one very heavy three grain german dark bread. Only the white italian bread showed a possible fractal structure after the squashing procedure, characterized by the dimension $2.81 \pm 0.06$, while the results for the others, respectively $D=2.95 \pm 0.06$ and $D=2.98 \pm 0.04$, for the dark and white sandwich breads, do not typify them as fractals. The conceptual problems, which resulted in a fractal dimension of $D=2.08 \pm 0.25$ for bread, found in an article in the literature, are analysed.
\end{abstract}

\section{Introdução}

A motivação que determinada tarefa possa despertar nos estudantes é indiscutivelmente uma componente importante no processo ensino-aprendizagem. Decorre daí que temas de maior evidência na mídia podem ser explorados com vantagem em atividades didáticas, sempre que isso esteja em acordo com os objetivos gerais colocados para o curso. Um tema em relativa evidência nos últimos anos se refere a estruturas fractais.

Alguns anos atrás, Marcelo A.F. Gomes, da Universidade Federal de Pernambuco, apresentou no American Journal of Physics [1] uma atividade interessante, que envolve o estudo de propriedades fractais em bolas de papel amassado. Esta atividade tem sido empregada já há alguns anos, com sucesso, no laboratório didático do Instituto de Física da USP(IFUSP). Ela é apli- cada como terceiro experimento, dentro de uma série destinada a salientar flutuações em medições [2, 3, 4] para ingressantes nos cursos de bacharelado de Física, Geofísica e Meteorologia (Física Experimental 1). Embora o tema seja estimulante, ficou evidente, ao longo dos anos, que o conceito do que seja um fractal está sujeito a confusões. De fato, os alunos ora o tomam de forma restrita como se o conceito implicasse numa auto-similaridade absoluta (que não se aplica aos papéis amassados) ou o associam simplesmente à porosidade do objeto.

À procura de situações experimentais análogas à coleção de papéis amassados, que pudessem ser utilizadas em questão de prova dentro do sistema avaliação empregado na disciplina [5], a equipe de professsores deparou-se, ademais, com uma interpretação incorreta do conceito de fractal aplicada a pão amas- 
sado, publicada numa das edições do Physics Teachers [6]. Chamou a atenção o valor indevidamente baixo, $D=2,08 \pm 0,25$, obtido para o pão, o qual, imediatamente, pode ser associado à expressão, dimensionalmente incorreta, empregada na análise. Examinando-se melhor o texto, verificou-se que, além deste problema, havia na publicação outros, associados à conceituação do fractal. Ilustrando a questão com dados tomados para três produtos de panificação, os autores do presente estudo publicaram uma nota na mesma revista [7], em que foi rebatida a informação incorreta, sem, entretanto, contarem com espaço suficiente para uma discussão mais ampla do conceito. Apresenta-se, aqui, essa discussão. O presente trabalho visa, além disso, dirimir uma dúvida que permaneceu sobre a fractalidade do objeto pão amassado, já que, com incertezas da ordem de $3 \%$, embora estas não excluíssem a dimensão $D=3,0$, era possível, ou até provável, que um estudo mais detalhado e preciso concluísse, por exemplo, que $D \sim 2,9$ para os pães.

Antes de mais nada, é necessário salientar que não é o fato de um objeto ser não-homogêneo ou poroso que o torna candidato a fractal; a não-homogeneidade deve seguir uma lei de formação, tal que ela se modifique conforme o tamanho do objeto [8]. Na próxima secção detalha-se essa noção. Finalmente, deve-se considerar que as idéias aqui discutidas podem ser estendidas a outras situações experimentais, por exemplo, enrolar, em espiral ou em formato de bolas, fios metálicos finos.

\section{I.1 A conceituação de fractais}

Segundo o trabalho pioneiro de Mandelbrot [9], os fractais são definidos como estruturas auto-similares, ou seja, aquelas que se apresentam da mesma forma, qualquer que seja a escala de fragmentação. Outras definições podem ser encontradas na literatura, algumas baseadas em propriedades de funções matemáticas [10]. Entretanto, está claro, hoje em dia, que a auto-similaridade pode ser entendida como propriedade média, do ponto de vista estatístico, não precisando se aplicar a todos os detalhes das estruturas $[8,11,12]$. Este é o caso mais comum na natureza e representa também alguns objetos fabricados pelo homem, em particular bolas de papel amassado. Um conceito interessante, neste contexto, é o da densidade fractal [11].

A diferença principal entre estruturas euclideanas e fractais, sob este ponto de vista, pode ser entendida pela análise da relação entre a massa do objeto $M$ e uma dimensão linear característica do mesmo, $r$. No caso das estruturas euclideanas, a proporcionalidade se dá com $r^{d}$, sendo $d$ um número natural, enquanto, para os fractais ela se dá com $r^{D}$, onde $D$ é um número não-inteiro. Isso significa que objetos fractais não possuem densidades volumétricas, superficiais ou lineares constantes, obedecendo, porém, a uma lei de formação tal que é possível definir uma densidade fractal, não constante, $\rho(r)$ [11]. As relações, a seguir, explicitam melhor o conceito.

Tome-se um objeto com simetria aproximadamente esférica, com massa $M=k r^{D}$ e obtenha-se $\rho(r)$ por considerações usuais para uma casca esférica de espessura $\delta r$ infinitesimal:

$\rho(r)=\lim _{\delta r \rightarrow 0} \frac{M(r+\delta r)-M(r)}{V(r+\delta r)-V(r)}=\frac{k(r+\delta r)^{D}-k r^{D}}{\frac{4}{3} \pi(r+\delta r)^{d}-\frac{4}{3} \pi r^{d}}$

$$
\rho(r)=\lim _{\delta r \rightarrow 0} C \frac{r^{D-1} \delta r+[\delta r]^{2}}{r^{d-1} \delta r+[\delta r]^{2}} \simeq C r^{D-d} .
$$

O atributo físico que faz com que a distribuição de massa de um objeto fractal obedeça a uma lei de potência (expressão (2)) é a ausência de uma escala ou comprimento característico. Assim, a lei matemática de formação é, em última análise, o reflexo de uma característica física apresentada por objetos fractais.

Na expressão (2) estão-se enfocando, de forma particular, esferas de raio $r$, mas considerações análogas poderiam ser feitas para objetos cúbicos, discos de raio $r$, etc.

Esse resultado extraordinário permite visualizar que, se forem tomadas cascas esféricas concêntricas, determinando sua densidade volumétrica média, esta será tanto menor quanto mais afastada do centro da esfera a casca estiver, já que para este tipo de estrutura $d \geq D$, e portanto $D-d \leq 0$. Por outro lado, em vez de olhar como se comporta a densidade dentro de uma única esfera, pode-se, de forma equivalente, analisar a densidade de várias esferas de mesma espécie, de raios diferentes. Nota-se imediatamente que, se $D=d$, a definição (Eq. (2)) se reduz à usual, da densidade volumétrica

$$
\rho(r)=C=\text { constante } .
$$

A expressão também mostra que, apresentar densidade não-constante, dependente da distância ao centro, é condição necessária, mas não suficiente, para o objeto ser considerado fractal. Assim, por exemplo, para relações $M \propto r^{1}$ ou $M \propto r^{2}$, isto é, se $D=1$ ou $D=2$, números naturais, para objetos tridimensionais, ou seja com $d=3$, obtêm-se respectivamente

$$
\rho(r)=\frac{C^{\prime}}{r^{2}} \quad(D=1) \quad e \quad \rho(r)=\frac{C^{\prime \prime}}{r} \quad(D=2)
$$

e estes objetos não são fractais. 
De fato, no primeiro caso, uma interpretação fácil é considerar um objeto feito de filamentos que se abrem, a partir de um centro, mas não se ramificam. Seria necessária a ramificação, segundo determinada lei de formação, para o objeto tornar-se um fractal. Um exemplo clássico de fractal embebido na dimensão $d=2$ é a folha de samambaia rendada ou depósitos metálicos crescidos sob certas condições eletroquímicas [12].

Uma das maneiras mais diretas de estudar as propriedades fractais de objetos é, portanto, analisar como se comporta a massa em função de alguma dimensão linear característica, segundo a hipótese

$$
M=k r^{D}
$$

Detalhando-se, tomando-se uma coleção de objetos de massas e tamanhos diferentes, mas fabricados, aparentemente, de modo similar, mede-se alguma dimensão linear (diâmetro, aresta, etc ...) e a massa de cada objeto, verificando-se, em seguida, se é possível definir uma relação entre as grandezas que apresente valores constantes para $k$ e $D$. Se, ainda, $D$ resultar em um número não-natural, está-se caracterizando esta coleção de objetos como fractais. A análise gráfica da Eq. (5) linearizada, $\log M=\log k+D \log r$, é a maneira mais direta de se obter a informação desejada, pois resulta em uma reta de coeficiente angular $D$, no caso da hipótese feita se demonstrar verdadeira.

\section{Procedimento Experimental}

O presente artigo estuda, através de uma coleção de dados mais extensa, medições mais controladas e empregando uma variedade maior de pães, se propriedades fractais podem ser atribuídas a este alimento tão comum, depois de amassá-lo. O experimento em si é de fácil execução e pode ser utilizado também como atividade didática rotineira.

O experimento consistiu em medir as massas e os diâmetros de bolas amassadas obtidas a partir de cubos de pão branco, de forma e italiano, e um tipo de pão preto multigrão alemão, bem denso. Os dois primeiros foram comprados em uma padaria, não-fatiados, enquanto o último é industrializado por Mestemacher e comercializado pela WICKBOLD ${ }^{\circledR} \quad$ (três grãos) e fatiado. O pão de forma foi obtido já sem casca e os outros tiveram a casca retirada. Os cubos foram amassados, sempre pela mesma pessoa e da mesma forma, tentando-se obter um objeto de forma esférica. Após amassadas, as bolas de pão foram deixadas para respirar por mais de 20 minutos antes de serem medidas, para garantir que todas fossem manipuladas já em sua forma de equilíbrio. A forma das bolas não é perfeita- mente esférica e há a presença de algumas rachaduras pela falta de ligamento da massa do pão.

A massa das bolas foi medida uma única vez com uma balança analítica com menor divisão de 0,0001 $\mathrm{g}$. Entretanto, dada a variação da massa da bola durante o processo de medida, provavelmente devido à evaporação da água da superfície, a incerteza estimada foi tomada como $0,001 \mathrm{~g}$. O diâmetro foi medido, com uma régua de metal calibrada, de menor divisão de 0,1 cm, em oito posições diferentes para se obter um valor representativo do diâmetro da bola. As medições dos diâmetros foram realizadas com a bola de pão sobre a régua, lendo-se na vertical os valores da escala da régua, com o cuidado de se evitar o efeito de paralaxe.

Os resultados obtidos para as grandezas massa e diâmetro das bolas são apresentados na Tabela 1. Conforme se nota, houve preocupação de não apenas colher uma grande quantidade de dados (de 8 a 12 bolas de tamanhos diferentes), como estender o intervalo de massas investigado. Para o pão de forma foram produzidas seis bolas de massa aproximadamente $6,5 \mathrm{~g}$ para se verificar a reprodutibilidade do processo de amassar o pão. Esse conjunto de bolas será referido, a partir de agora, como grupo controle. A bola de pão de forma de massa $103 g$ foi medida duas vezes: uma logo após o processo de amassar e a outra cerca de 20 minutos depois para verificar o efeito de sua respiração. Esse efeito produziu uma variação de diâmetro de 5,63 $\pm 0,07$ $\mathrm{cm}$ para $5,71 \pm 0,07 \mathrm{~cm}$, valores estatisticamente equivalentes.

\section{Análise dos Resultados}

A análise dos dados é referenciada na relação $M=K \phi^{D}$ (Eq. (5), escrita para o diâmetro, $\phi=2 r$, com $K=k / 2^{D}$ ), já que as medições são dos diâmetros das bolas. Para linearização da curva que representa os dados experimentais, os alunos normalmente utilizam a logaritmização, construindo gráfico em papel di-log comercial. Este apresenta três décadas no eixo horizontal e apenas duas no vertical; por conveniência opta-se, então, por transformar a Eq. (5) em

$$
\phi=\left(\frac{M}{K}\right)^{\frac{1}{D}}
$$

Desta forma,

$$
\log \phi=\frac{1}{D} \log \frac{1}{K}+\frac{1}{D} \log M
$$

sendo, então,

$y=b+a x, \operatorname{com} y=\log \phi, x=\log M, a=\frac{1}{D} \mathrm{e}$ $b=\frac{1}{D} \log \frac{1}{K}$. 
Tabela 1: Massa $M$ e diâmetro médio $\phi$ das bolas de pão obtidas por amassamento. Entre parênteses se encontram indicadas as incertezas associadas aos valores médios dos diâmetros. A incerteza no valor das massas foi estimada como $0,001 \mathrm{~g}$, exceto para as duas últimas massas de pão preto, para os quais foi tomada como $0,01 \mathrm{~g}$, porque havia a possibilidade da bola soltar pequenos fragmentos durante as medições.

\begin{tabular}{|c|c|c|c|c|c|}
\hline \multicolumn{2}{|c|}{$\overline{P \text { Pão de forma }}$} & \multicolumn{2}{|c|}{ Pão preto três grãos } & \multicolumn{2}{|c|}{ Pão italiano } \\
\hline$M(g)$ & $\bar{\phi}(\mathrm{cm})$ & $M(g)$ & $\bar{\phi}(\mathrm{cm})$ & $M(g)$ & $\bar{\phi}(\mathrm{cm})$ \\
\hline 0,167 & $0,68 \pm 0,05$ & 0,407 & $0,90 \pm 0,05$ & 0,149 & $0,63 \pm 0,05$ \\
\hline 0,196 & $0,73 \pm 0,05$ & 0,686 & $1,08 \pm 0,05$ & 0,805 & $1,14 \pm 0,06$ \\
\hline 0,266 & $0,83 \pm 0,06$ & 1,835 & $1,49 \pm 0,06$ & 2,186 & $1,60 \pm 0,06$ \\
\hline 0,426 & $0,93 \pm 0,05$ & 2,055 & $1,54 \pm 0,07$ & 5,455 & $2,28 \pm 0,06$ \\
\hline 0,742 & $1,13 \pm 0,05$ & 6,261 & $2,21 \pm 0,06$ & 5,635 & $2,21 \pm 0,07$ \\
\hline 1,369 & $1,34 \pm 0,06$ & 10,545 & $2,69 \pm 0,08$ & 10,927 & $2,69 \pm 0,09$ \\
\hline 2,715 & $1,69 \pm 0,06$ & 19,660 & $3,26 \pm 0,07$ & 37,565 & $4,41 \pm 0,07$ \\
\hline 3,313 & $1,78 \pm 0,05$ & 25,40 & $3,55 \pm 0,10$ & 51,148 & $5,01 \pm 0,11$ \\
\hline 6,420 & $2,18 \pm 0,06$ & 42,71 & $4,35 \pm 0,06$ & & \\
\hline 6,437 & $2,18 \pm 0,07$ & & & & \\
\hline 6,456 & $2,26 \pm 0,07$ & & & & \\
\hline 6,470 & $2,21 \pm 0,05$ & & & & \\
\hline 6,489 & $2,28 \pm 0,06$ & & & & \\
\hline 6,510 & $2,23 \pm 0,05$ & & & & \\
\hline 12,090 & $2,86 \pm 0,06$ & & & & \\
\hline 29,558 & $3,83 \pm 0,06$ & & & & \\
\hline 102,606 & $5,71 \pm 0,07$ & & & & \\
\hline
\end{tabular}

Tabela 2: Resultados obtidos para a dimensão fractal $D$ e constante $K$, de acordo com a Eq.(6), para as bolas de pão amassado.

\begin{tabular}{||c|c|c||}
\hline \hline tipo de pãa & dimensãa fractal D & constante $K\left(\mathrm{gcm}^{-D}\right)$ \\
\hline Pão de forma & $2,98 \pm 0,04$ & $0,570 \pm 0,018$ \\
Pão preto três grãos & $2,95 \pm 0,06$ & $0,579 \pm 0,030$ \\
Pão italiano & $2,81 \pm 0,06$ & $0,578 \pm 0,031$ \\
\hline \hline
\end{tabular}

*neste caso, tomou-se também a relação que resulta de $D=3$.

Tabela 3: Resultados obtidos para a dimensão fractal D e constante $\mathrm{K}$ para as bolas de pão branco, pão preto e bolo do trabalho de Amaku et al. [7].

\begin{tabular}{||c|c|c||}
\hline \hline tipo de pãa & dimensão fractal $D$ & constante $K\left(\mathrm{gcm}^{-D}\right)$ \\
\hline Pão de forma (PANCO $\left.{ }^{\circledR}\right)$ & $2,91 \pm 0,12$ & $0,630 \pm 0,038$ \\
Pão preto & $2,87 \pm 0,07$ & $0,597 \pm 0,028$ \\
Bolo & $2,81 \pm 0,11$ & $0,635 \pm 0,039$ \\
\hline \hline
\end{tabular}

Assim, se o modelo adotado para a relação entre massa e diâmetro das bolas de pão amassado (Eq. (6)) for adequado, o gráfico de $\log \phi$ versus $\log M$ deve ser uma reta e a dimensão fractal $D$ pode ser obtida diretamente pelo inverso da inclinação desta.
Os gráficos linearizados, $\log \phi \times \log M$ para os três tipos de pão são apresentados na Fig. 1 e pode-se apreciar que os dados são bem representados por retas. Chama-se a atenção para o fato de que, em particular para o pão branco de forma, há informações sobre 
bolas de pão cuja massa varia de um fator maior do que 500. Para esse pão, foi analisado um conjunto de seis bolas fabricadas a partir de cubos de pão cortados inicialmente de forma muito semelhante para resultar em pedaços de $\sim 6,5 g$ (grupo controle).
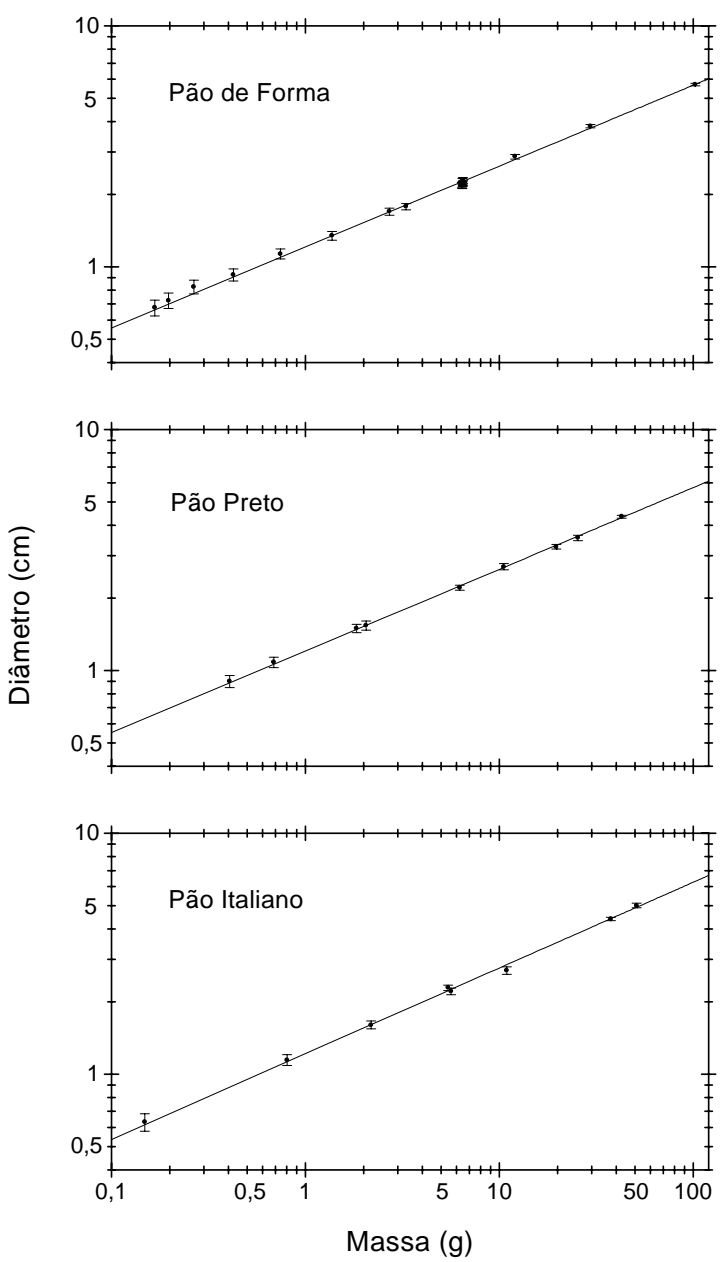

Figura 1. Diâmetro das bolas de pão amassado em função da massa, em escala logarítmica, para os três tipos de pães analisados.

Fazendo-se um ajuste, pelo método dos mínimos quadrados, entre resultados experimentais e a Eq. (7), foram obtidos os valores de $D$ e $K$ da Tabela 2. A Fig. 2, que apresenta uma ampliação do gráfico mostrado na Fig. 1 na região do grupo controle, demonstra que o processo de amassamento é bastante reprodutível, mas que é difícil produzir bolas que possam, de fato, ser consideradas de mesma massa.

Na Tabela 3 são retomados os valores obtidos anteriormente pelos autores [7] para um pão de forma (marca PANCO ${ }^{\circledR}$ ), semelhante ao analisado na seção II, para um pão preto comum (marca PULLMAN ${ }^{\circledR}$ ), menos denso do que o da WICKBOLD ${ }^{\circledR}$, e para um bolo de coco (marca PANCO ${ }^{\circledR}$ ). Um número menor de pontos nos gráficos e um processo de medição menos controlado haviam, então, levado a incertezas maiores do que as obtidas no presente trabalho. Nota-se que o processo é reprodutível e que, em particular, o pão de forma amassado, o qual foi retomado no presente trabalho, tem dimensão compatível com $D=3$. Ambos os pães pretos, embora originalmente de consistência muito diferente, resultaram em bolas com características muito semelhantes a esferas euclideanas, já que, pelas Tabelas 2 e 3, são compatíveis com $D=3$ dentro de dois desvios-padrão. O único tipo de pão que não admite $D=3$, dentro de três desvios-padrão, é o tipo italiano.

Finalmente, observando-se os valores de $K$ nas Tabelas 2 e 3, nota-se que são bastante próximos. No caso de esferas euclideanas, com $D=3$ (situação muito próxima à observada experimentalmente), resulta $K=\pi \rho / 6$, com $\rho$ a densidade volumétrica, constante. Percebe-se, pois, que todas as bolas de pão amassado apresentam densidade volumétrica parecida, um pouco acima da densidade da água. Isso é notável, pois partiu-se de cubos de densidades volumétricas originalmente muito diferentes, sendo os pães brancos os mais leves e o pão preto três grãos, de longe, o mais denso. A Tabela 4 compara a densidade volumétrica dos pães, antes de serem amassados, com a densidade volumétrica que resulta do valor de $K$ obtido experimentalmente, considerando-se $D=3$, para todos. No processo de amassamento, aparentemente, chegou-se a um tipo de agregamento típico dos constituintes usuais dos pães.

\section{Discussão e Conclusão}

Atividades motivadoras, centradas em tópicos modernos, encerram em si a possibilidade de induzir ou reforçar conceitos errôneos. Sabe-se, por outro lado, que pré-conceitos incorretos, mais do que ausência de qualquer informação, dificultam ações pedagógicas posteriores. No transcorrer da disciplina de Física Experimental 1, foram detectadas dificuldades conceituais associadas a fractais na atividade [4] que estuda bolas de papel amassado. A preocupação foi intensificada quando se verificou que havia publicação, na conceituada revista Physics Teachers [6], a qual, ao trabalhar com pães, evidenciava erros conceituais do mesmo tipo daqueles verificados com alunos do IFUSP, 
além de outros. Como conseqüência, aquele trabalho [6] colocava a público uma dimensão fractal extremamente baixa ( $D=2,08 \pm 0,25)$ para um pão branco amassado, objeto tridimensional. Os autores do presente estudo tiveram a oportunidade de retificar a informação em nota curta publicada na mesma revista [7]. O equívoco estava relacionado a três distorções conceituais, aparentemente comuns, e para as quais é necessário atentar durante a aula:

(i) é impossível caracterizar um único objeto como frac- tal, sem analisá-lo por partes;

(ii) não é pelo fato de um objeto ser pouco homogêneo, poroso ou constituído por filamentos que ele é, por natureza, candidato a ser fractal; e

(iii) todas as expressões físicas, mesmo em áreas novas ou modernas, precisam adequar-se a uma análise dimensional consistente, para representarem resultados invariantes por mudança de unidade. Apenas este último aspecto pôde ser abordado na nota retificadora $[7]$.

Tabela 4: Densidade volumétrica dos pães antes de serem amassados, em comparação com a densidade volumétrica que resulta do valor $K$ obtido experimentalmente depois de amassados, considerando $D=3,0$.

\begin{tabular}{||c|c|c||}
\hline \hline tipo de pãa & $\begin{array}{c}\text { densidade do pão } \\
\text { antes do amassamento } \\
\left(\mathrm{gcm}^{-3}\right)\end{array}$ & $\begin{array}{c}\text { densidade do pãó } \\
\text { após o amassamento } \\
\left(\mathrm{gcm}^{-3}\right)\end{array}$ \\
\hline Pão de forma & $0,140 \pm 0,006$ & $1,070 \pm 0,013$ \\
Práo preto três grãos & $0,698 \pm 0,025$ & $1,087 \pm 0,023$ \\
Pão italiano & $0,261 \pm 0,009$ & $\sim 1,066 \pm 0,027^{*}$ \\
Pão de forma (PANCO $\left.{ }^{\circledR}\right)$ & $0,184 \pm 0,008$ & $1,203 \pm 0,073$ \\
Pão preto & $0,253 \pm 0,007$ & $1,140 \pm 0,053$ \\
Bolo & $0,402 \pm 0,014$ & $1,213 \pm 0,074$ \\
\hline \hline
\end{tabular}

*neste caso, tomou-se também a relação que resulta de $D=3$.

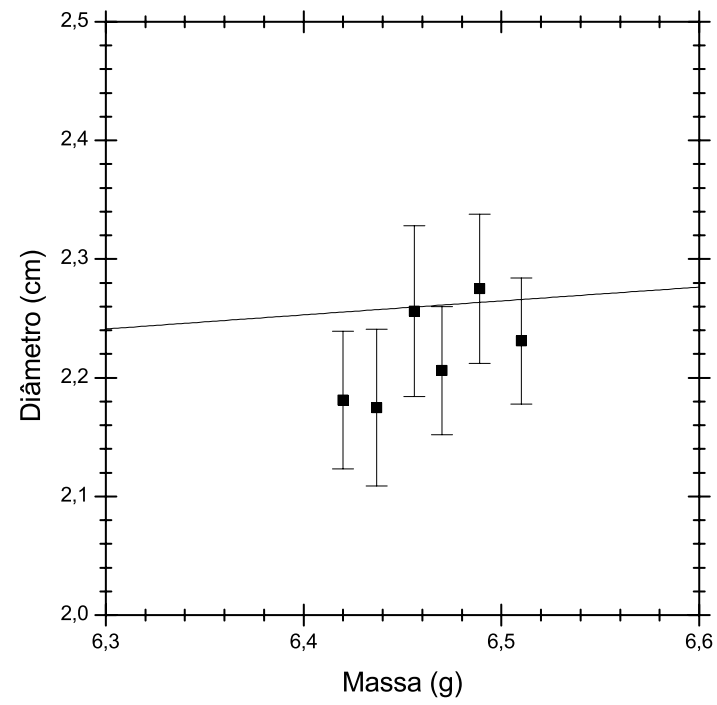

Figura 2. Diâmetro das bolas de pão de forma amassado em função da massa com a escala expandida na região do grupo controle. A curva do gráfico corresponde à função dada pela Eq. (6), ajustada ao conjunto total dos 17 valores experimentais, cujos parâmetros se encontram na Tabela 2 .

Além disso, um aspecto interessante a ser mencionado diz respeito à porosidade como critério para definir um objeto como fractal. Um objeto que apresente poros com tamanho característico regular ou aleatório não deve apresentar estrutura fractal. Por outro lado, a fractalidade deve resultar da ausência de um comprimento característico dos poros, relacionada, como já mencionamos na Seção I, a uma lei de formação.

Aos conjuntos de bolas de pão fabricado amassado, a partir de vários tipos de produtos de padaria, tanto os utilizados no trabalho anterior [7], quanto os do presente estudo, foi aplicada uma análise análoga àquela apresentada como proposta de experiência didática em 1987 por M.A.F. Gomes [1], em relação a bolas de papel amassado. Este último tipo de atividade didática tem sido empregada na disciplina de Física Experimental 1 do Instituto de Física da USP desde 1995, com resultados que confirmam o valor $D \sim 2,5$ para bolas feitas com papéis com densidades superficiais próximas à do papel sulfite usual. Do ponto de vista instrucional, dentro da disciplina do IFUSP, a experiência dos papéis amassados visa a reforçar o conceito de desviopadrão para uma distribuição de resultados de medições (por repetição de um número grande de medições em uma das bolas) e demonstrar a vantagem de análises 
linearizadas através de transformações de variáveis. A atividade vem confirmando sua componente motivacional, mas demonstrou, também, que o conceito do que seja um fractal é de difícil assimilação. Há, pois, necessidade de procurar situações complementares, que favoreçam a discussão das expectativas incorretas, com os estudantes.

Com o estudo mais completo aqui apresentado concluiu-se que, se forem amassados pães de diversos tipos, obter-se-ão esferas praticamente euclideanas, mesmo para pães originalmente muito pouco homogêneos, como os pães pretos ou bastante porosos, como os pães de forma. O pão branco do tipo italiano é o único pão, dos investigados, que pode resultar em bolas com características de fractal. Nota-se que esse apresenta certa elasticidade ao toque e pode ser que, em função desta, acabe por acomodar-se de forma diferente perto das superfícies.

A experiência dos pães amassados pode, então, ser explorada como um contraponto não fractal à experiência, mais conhecida, das bolas de papel amassado [1], para as quais a estrutura fractal é verificada em média. Sugere-se, por exemplo, que metade dos grupos de alunos de cada classe trabalhe com uma ou outra das situações: pão, de diversos tipos, e papel. Isso, após confrontação dos resultados dentro de suas incertezas, demonstra a necessidade de considerar as precisões das medidas para informar se $D \neq 3,0$ ou não. Outra possibilidade é solicitar que metade dos estudantes da classe molhem os papéis antes de amassá-los, o que também resulta em esferas praticamente euclideanas.

É importante, ainda, salientar que as idéias aqui desenvolvidas podem ser aplicadas a outras situações experimentais, por exemplo, enrolar, alternativamente, fios metálicos ou barbante, seja em forma de espirais planas, seja em forma de bolas.
Agradecimentos

M Amaku agradece o apoio financeiro da FAPESP e P R Pascholati o apoio financeiro do CNPq.

\section{References}

[1] M A F Gomes, Am J Phys 55;649-50(1987)

[2] L B Horodynski-Matsushigue et al., Planning an introductory laboratory for physics freshmen: Ten years of growing understanding at São Paulo University, in Atas da Conferência Interamericana sobre Educação em Física, Canela, RS, 3 a 7 de Julho de 2000, editada por Marco Antonio Moreira, Instituto de Física da Universidade Federal do Rio Grande do Sul, Porto Alegre, 2000, CD-ROM.

[3] L B Horodynski-Matsushigue et al., Incertezas Experimentais: É possivel Convencer os Alunos de sua Utilidade? XIII Simpósio Nacional de Ensino de Física, 25 a 29-01-1999, Brasília, DF, painel, in Caderno de Resumos e Programação, pág. 18

[4] J H Vuolo et al., Física Experimental I, apostila, IFUSP, 2001, São Paulo.

[5] L B Horodynski-Matsushigue et al., Assessing in the laboratory: can it be significant?, in Atas da Conferência Interamericana sobre Educação em Física, Canela, RS, 3 a 7 de Julho de 2000, editada por Marco Antonio Moreira, Instituto de Física da Universidade Federal do Rio Grande do Sul, Porto Alegre, 2000, CDROM.

[6] D H Esbenshade Jr, Phys Teach 29;236-6(1991)

[7] M Amaku, L B Horodynski-Matsushigue e P R Pascholati, Phys Teach 37;480-1(1999)

[8] J Feder, Fractals, Plenum Press, New York, 1988

[9] B B Mandelbrot, The Fractal Geometry of Nature, Freeman, San Francisco, 1982

[10] M F Barnsley, Fractals Everywhere, $2^{\underline{a}}$ edição, Academic Press, Boston, 1993.

[11] R Orbach, Science 231;814-9(1986)

[12] V Talanquer e G Irazoque, Phys Teach 31; 72-78(1993) 